\title{
PEDIATRICS \\ GENOMIC STUDIES OF SEX DEVELOPMENT
}

New research has led to the identification of gene variants that might contribute to disorders of sexual development (DSDs).

The first study, by a research group at Baylor College of Medicine in the USA, describes several copy number variants found in children with idiopathic DSDs. DNA was extracted from the blood of 116 children born with ambiguous genitalia, cryptorchidism or hypospadias and analyzed on a comparative genomic hybridization microarray. The researchers were looking for clinically relevant submicroscopic chromosomal rearrangements, too small to be detected by traditional methods. Accordingly, de novo microdeletions and microduplications were found in 37 children, $74.2 \%$ of which were not detected during karyotype analysis. Of particular note, variants were found for ambiguous genitalia on 9p24.3, a region that encompasses the testis-specific gonad-determining gene DMRT1.

The second study, performed at Radboud Nijmegen Medical Centre in The Netherlands, details the identification of $D G K K$ as a major risk gene for hypospadias. Using pooled DNA from 436 affected males and 494 control individuals of European descent, researchers carried out a genome-wide association study to find single nucleotide polymorphisms (SNPs) associated with hypospadias. After refining their results according to allele frequencies and quality control criteria, researchers were left with 20 SNPs, seven of which were located in DGKK on the $\mathrm{X}$ chromosome. Individual genotyping and replication studies in two other patient cohorts confirmed the association. Finally, polymerase chain reaction experiments detected expression of $D G K K$ in the preputial skin of boys affected by hypospadias and of healthy controls.

Sarah Payton

Original articles Tannour-Louet, M. et al. Identification of de novo copy number variants associated with human disorders of sexual development. PLoS One 5, e15392 (2010) | van der Zanden, L. F. et al. Common variants in $D G K K$ are strongly associated with risk of hypospadias. Nat. Genet. doi:10.1038/ng.721 